\title{
Effective Adsorption and Sensitive Detection of Cr(VI) by Chitosan/Cellulose Nanocrystals Grafted with Carbon Dots Composite Hydrogel
}

\author{
Hua Zeng ${ }^{1}$, Zhiyuan Hu ${ }^{1}$, Chang Peng ${ }^{2}$, Lei Deng ${ }^{2}$ and Suchun Liu ${ }^{1, *}$ \\ 1 College of Food Science and Technology, Hunan Agricultural University, Changsha 410128, China; \\ zh@hunau.edu.cn (H.Z.); huzhiyuan@hncu.edu.cn (Z.H.) \\ 2 School of Chemistry and Materials Science, Hunan Agricultural University, Changsha 410128, China; \\ pengchang@hunau.edu.cn (C.P.); leideng@hunau.edu.cn (L.D.) \\ * Correspondence: liusc@hunau.net
}

Citation: Zeng, H.; Hu, Z.; Peng, C.; Deng, L.; Liu, S. Effective Adsorption and Sensitive Detection of $\mathrm{Cr}(\mathrm{VI})$ by Chitosan/Cellulose Nanocrystals Grafted with Carbon Dots Composite Hydrogel. Polymers 2021, 13, 3788. https://doi.org/10.3390/ polym 13213788

Academic Editor: Ki Hyun Bae

Received: 9 October 2021

Accepted: 29 October 2021

Published: 1 November 2021

Publisher's Note: MDPI stays neutral with regard to jurisdictional claims in published maps and institutional affiliations.

Copyright: (c) 2021 by the authors. Licensee MDPI, Basel, Switzerland. This article is an open access article distributed under the terms and conditions of the Creative Commons Attribution (CC BY) license (https:/ / creativecommons.org/licenses/by/ $4.0 /)$.

\begin{abstract}
Due to its lethal effect on the human body and other creatures, $\mathrm{Cr}(\mathrm{VI})$ ions have attained widespread public attention, and an effective adsorbent for removing $\mathrm{Cr}(\mathrm{VI})$ ions is vital. Chitosan (CS)/cellulose nanocrystals grafted with carbon dots (CNCD) composite hydrogel with strong sorption ability and sensitive detection ability for $\mathrm{Cr}(\mathrm{VI})$ was formed. The cellulose nanocrystals $(\mathrm{CN})$ offered a natural skeleton for assembling 3D porous structures, and then improved the sorption ability for $\mathrm{Cr}(\mathrm{VI})$; moreover, carbon dots (CD) acted as a fluorescent probe for $\mathrm{Cr}(\mathrm{VI})$ and provided $\mathrm{Cr}(\mathrm{VI})$ adsorption sites. With a maximum adsorption capacity of $217.8 \mathrm{mg} / \mathrm{g}$, the CS/CNCD composite hydrogel exhibited efficient adsorption properties. Meanwhile, with a detection limit of $0.04 \mu \mathrm{g} / \mathrm{L}$, this hydrogel was used for selective and quantitative detection of $\mathrm{Cr}(\mathrm{VI})$. The determination of $\mathrm{Cr}(\mathrm{VI})$ was based on the inner filter effect (IFE) and static quenching. This hydrogel retained its effective adsorption ability even after four repeated regenerations. Furthermore, the economic feasibility of the CS/CNCD composite hydrogel over activated carbon was confirmed using cost analysis. This study provided one new method for producing low-cost adsorbents with effective sorption and sensitive detection for $\mathrm{Cr}(\mathrm{VI})$.
\end{abstract}

Keywords: chitosan; cellulose nanocrystals; carbon dots; adsorption; hexavalent chromium

\section{Introduction}

Due to rapid industrial development, water contamination by heavy metals has become more and more serious [1]. To remove heavy metals, many traditional methods, such as chemical precipitation, membrane filtration, photocatalytic degradation, and adsorption are currently used [2]. Adsorption is regarded as one of the most promising approaches in these methods. As a result, creating an efficient adsorbent is essential.

CS has received widespread attention as an adsorbent for heavy metal ions due to its low cost and great adsorption potentials [3]. CS is blended with other materials leading to improved sorption performance. Pavithra et al. formed a surface-tailored chitosan/orange peel composite hydrogel to remove $\mathrm{Cr}(\mathrm{VI})$ and $\mathrm{Cu}(\mathrm{II})$ ions from synthetic wastewater, and then measured the adsorption capacity for these two ions [4]. Hao et al. prepared N-carboxymethyl chitosan hydrogel, analyzed its morphology and structure, studied the influencing factors of adsorption performance, and discussed the intrinsic mechanism of the adsorption process [5]. The adsorption thermodynamics, kinetics, and adsorption mechanism of a composite chitosan-glucose hydrogel for sorption of $\mathrm{Co}^{2+}$ ion were discussed, and the adsorption capacity of $202 \mathrm{mg} / \mathrm{g}$ for $\mathrm{Co}^{2+}$ was demonstrated [6]. Although researchers have made great efforts, improving the adsorption capacity for heavy metals by the chitosan-based material is still a challenge. A general solution would be to introduce nanoparticles with excellent properties in order to increase adsorption 
capacity [7]. In this regard, we propose introducing cellulose nanocrystals (CN). Firstly, $\mathrm{CN}$ can serve as a scaffold around which a network structure can form. Second, CN can provide a large number of hydroxyl and carbonyl functional groups as heavy metal sorption sites [8]. Therefore, CS/CN composite hydrogel has great potentiality in the development of novel adsorbents.

Carbon-based fluorescent nanomaterials, such as carbon dots (CD), have recently stimulated the interest of researchers due to their unique optical and nontoxic properties $[9,10]$. Many studies have shown that CDs can be used to detect $\mathrm{Cr}(\mathrm{VI})$ optically [11,12]. However, some issues remain in sensor probe application, such as $\mathrm{CD}$ agglomeration with increasing detection and storage time [13]. One possible solution to overcome this problem is to graft with other materials, such as $\mathrm{CN}$ [14].

In this article, we aimed to create chitosan/CN grafted with CD (CS/CNCD) composite hydrogel for $\mathrm{Cr}(\mathrm{VI})$ detection and sorption. This hydrogel's morphology, structure, fluorescence properties, adsorption, and detection characteristics were investigated. In addition, the sorption interaction between $\mathrm{Cr}(\mathrm{VI})$ and this hydrogel was confirmed. The economic analysis of this hydrogel was also explored.

\section{Materials and Methods}

\subsection{Materials}

CS with a deacetylation degree of ca. 90\% (J\&K Scientific Ltd., Beijing, China). 2,2,6,6-Tetramethylpiperidinooxy (TEMPO) (98\%, Aladdin, Shanghai, China), 1-(3Dimethylaminopropyl)-3-ethylcarbodiimide hydrochloride (EDC) $(98 \%$, Aladdin, Shanghai, China), Citric acid (99.8\%, Aladdin, Shanghai, China), Ethylenediamine (98\%, Aladdin, Shanghai, China), N-Hydroxysuccinimide (NHS) (98\%, Aladdin, Shanghai, China), and Glutaraldehyde (GD) (50\% in $\mathrm{H}_{2} \mathrm{O}$, Aladdin, Shanghai, China).

\subsection{Synthesis of $C N$}

As previously reported, the $\mathrm{CN}$ was produced using never-dried bleached wood pulp [15]. Bleached Wood pulp (Jin Yi Inc., Shanghai, China) (2.0 g), NaBr (Aladdin, Shanghai, China) (0.2 g), and 2,2,6,6-Tetramethylpiperidinooxy (TEMPO, $0.04 \mathrm{~g}$ ) were mixed in water $(200 \mathrm{~g})$. The mixture was then thoroughly mixed with an aqueous solution of $\mathrm{NaClO}$ (Aladdin, Shanghai, China) ( $6 \mathrm{~mL}, 5 \%$ chlorine solution). The $\mathrm{pH}$ was maintained at 10, and this oxidation lasted for $24 \mathrm{~h}$. Subsequently, the mixture was washed with water. Six passes through a microfluidizer (M110P, Microfluidics Corp., Newton, MA, USA) were made with the obtained mixture. Subsequently, the $\mathrm{CN}$ suspension was prepared.

\subsection{Synthesis of $C D$}

The water-dispersible CD was synthesized and purified by Zhu et al. [16]. Citric acid $(2.1 \mathrm{~g})$ and Ethylenediamine $(268 \mu \mathrm{L})$ were dissolved in water $(20 \mathrm{~g})$. The mixture was placed in a Teflon reaction vessel (Yi Chuang company, Xi'an, China), sealed, and autoclaved for $5 \mathrm{~h}$ at $300{ }^{\circ} \mathrm{C}$. The autoclave was then cooled. To remove excess reactants, the solution was dialyzed in a dialysis bag (500-1000 D) (Fisher Scientific, Pittsburgh, PA, USA) against water for three days. The CD could be obtained within this dialysis bag. High-resolution TEM images of the CD are provided in Figure S1.

\subsection{Synthesis of $C N C D$}

The CN suspension (10 g, $1.0 \mathrm{wt} \%$ ) was activated with 1-(3-Dimethylaminopropyl)-3ethylcarbodiimide hydrochloride (EDC, $20 \mathrm{mg}$ ) and N-Hydroxysuccinimide (NHS, $16 \mathrm{mg}$ ) under vigorous stirring at $\mathrm{pH} 5$ for $15 \mathrm{~min}$. Further, a CD solution $(10 \mathrm{~mL}, \mathrm{pH} 9.2$, phosphate buffer (Aladdin, Shanghai, China)) containing various mass fractions $(0.02 \mathrm{wt} \%, 0.04 \mathrm{wt} \%$, $0.06 \mathrm{wt} \%, 0.08 \mathrm{wt} \%, 0.10 \mathrm{wt} \%$, and $0.12 \mathrm{wt} \%$ ) was added. For $12 \mathrm{~h}$, the coupling reaction was carried out with mild agitation at $25^{\circ} \mathrm{C}$. The resulting suspension was washed and dialyzed against water. Since the mass ratio of $\mathrm{CN}$ and $\mathrm{CD}$ could affect the fluorescence behavior of the $\mathrm{CNCD}$, it was further optimized using fluorescent intensity as the objective [17]. The 
optimal mass fraction of CD was determined to be $0.10 \mathrm{wt} \%$, as shown in Figure S2. The suspension was prepared and named $\mathrm{CNCD}$, using the optimized experimental conditions.

\subsection{Fabrication of CS/CNCD Composite Hydrogel}

$2 \mathrm{~g}$ of CS powder was dissolved in a beaker containing $98 \mathrm{~g}$ of $2 \%$ acrylic acid solution (Aladdin, Shanghai, China). Subsequently, CNCD suspension ( $5 \mathrm{~g}$ ) with different mass fractions $(0.2 \mathrm{wt} \%, 0.4 \mathrm{wt} \%, 0.6 \mathrm{wt} \%, 0.8 \mathrm{wt} \%$, and $1.0 \mathrm{wt} \%)$ was well dispersed into this solution through ultrasonic dispersion. GD $(25 \mathrm{~mL}, 5 \mathrm{wt} \%)$ was then added. After $2 \mathrm{~h}$ of stirring, the mixture was heated to $60{ }^{\circ} \mathrm{C}$, and was heat-preserved for $2 \mathrm{~h}$. Since the mass ratio of CS and CNCD in the prepared hydrogel could affect its sorption behavior, it was further optimized with maximum adsorption capacity as the aim [18]. As shown in Figure S3, the optimal mass fraction of CNCD was identified to be $0.8 \mathrm{wt} \%$. Using the optimized experimental conditions, the hydrogel was prepared. The obtained hydrogel was washed with water continuously to remove excess reactants before being cut into small discs, and labelled as CS/CNCD composite hydrogel for further testing. Furthermore, the blank hydrogel was created using the same method, with the exception that the CNCD was not introduced.

\subsection{Batch Sorption Experiment}

The sorption behaviors (effect of $\mathrm{pH}$, temperature, contact time and initial concentration, and reusability) of the CS/CNCD composite hydrogel and the blank hydrogel were investigated by placing $3.0 \mathrm{~g}$ of these two adsorbents in different flasks containing $100 \mathrm{~mL} \mathrm{Cr}(\mathrm{VI})$ solution, and shaking the flasks well. The $\mathrm{pH}$ was varied from 1 to 6 , and the temperature varied in the range of $15^{\circ} \mathrm{C}-45^{\circ} \mathrm{C}$. The effects of contact time $(5-160 \mathrm{~min})$ were tested. The concentration varied in the range of $20-120 \mathrm{mg} / \mathrm{L}$. All of the experiments were averaged and repeated three times, with only the mean values displayed. The maximum deviation for duplicates was typically less than $5 \%$.

\subsection{Instruments}

Scanning electron microscopy image was taken using a Quanta 250 FEG scanning electron microscope (FEI, Hillsboro, MI, USA). Fourier transform infrared spectra (FTIR) were collected via a NEXUS 870 spectrometer (Nicolet, Waltham, MA, USA). Fluorescence spectrum was obtained via a RF-5301PC luminescence spectrometer (Shimadzu, Kyoto, Japan). UV-visible spectrum was obtained via a 3100 UV-vis spectrophotometer (Shimadzu, Kyoto, Japan). X-ray photoelectron spectra (XPS) were measured using a PHI-5000 spectrometer (Ulvac-Phi, Kanagawa, Japan). X-ray diffraction (XRD) spectra were taken using a D8 ADVANCE diffractometer (Bruker AXS, Karlsruhe, Germany). Contact angle was determined using a Phoenix 300 contact angle analyzer (SEO Co. Ltd., Ansung, South Korea).

\section{Results}

\subsection{Schematic Diagram}

Firstly, we should re-examine our previous experiments. In this study, $\mathrm{CN}$ and $\mathrm{CD}$ were two additional elements, as shown in Figure 1. CNCD was first prepared by covalent bonding between $\mathrm{CN}$ and $\mathrm{CD}$. Secondly, CNCD was combined with CS. Lastly, crosslinking took place in CS chains for constructing CS/CNCD composite hydrogel. 


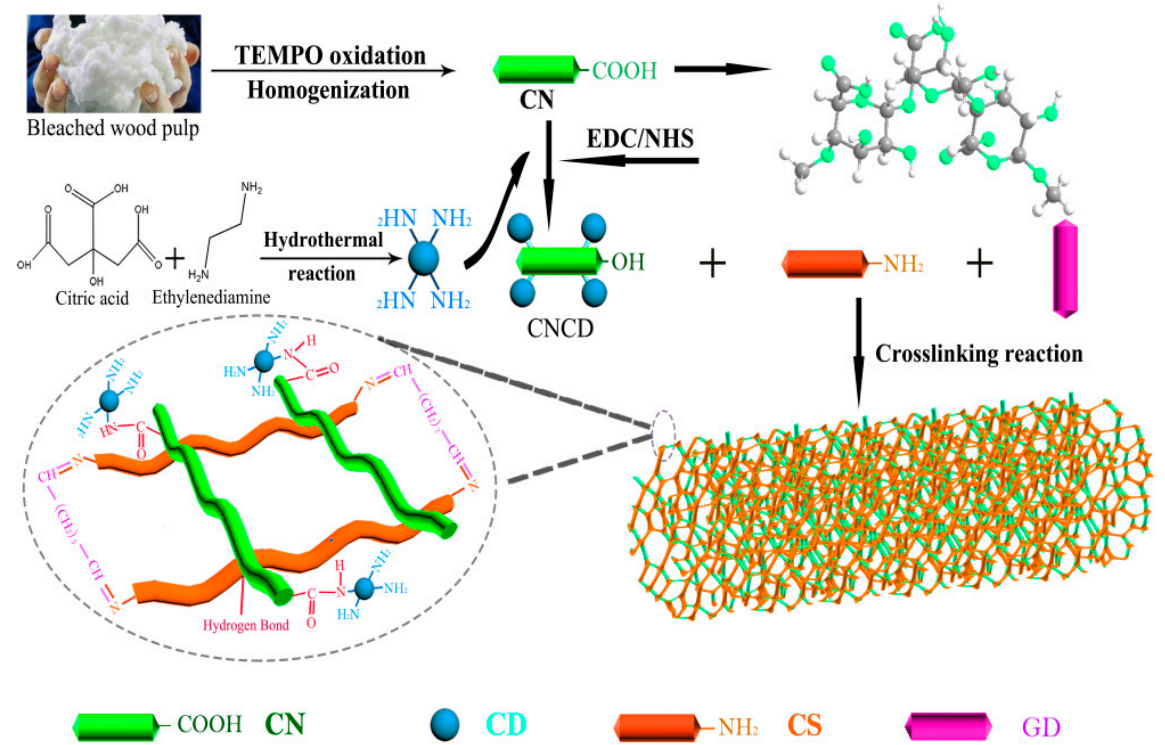

Figure 1. Schematic diagram of the synthesis process.

\subsection{Characterization}

Figure $2 \mathrm{a}, \mathrm{b}$ show the microstructures of the CS/CNCD composite hydrogel. This hydrogel had a 3D porous structure, which improved swelling ability (swelling ratio of $129 \mathrm{~g} / \mathrm{g}$ for deionized water at $25^{\circ} \mathrm{C}$ ). Using the crosslinking reaction, a 3D porous structure was required, and CD was wrapped [19]. Pore characteristics of the CS/CNCD composite hydrogel were evaluated in Figure S4, and the BET surface area and average pore width were $16.76 \mathrm{~m}^{2} / \mathrm{g}$ and $6.375 \mathrm{~nm}$, respectively. Moreover, the contact angle of $17.7^{\circ}$ showed the hydrophilicity of the CS/CNCD composite hydrogel [20].
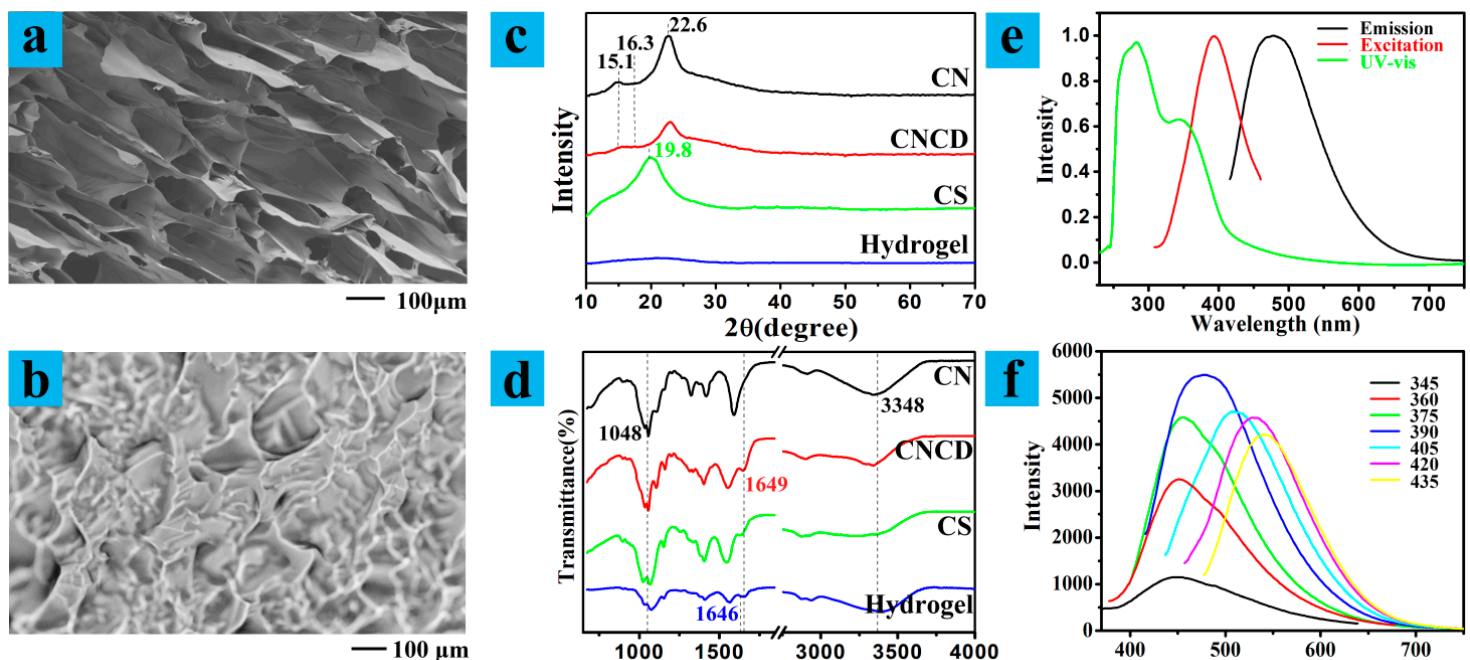

d
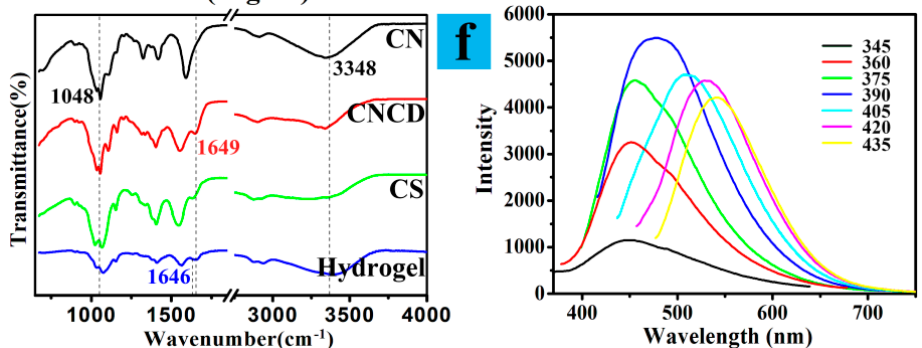

Figure 2. (a,b) SEM micrograph and optical microscope photo; (c) XRD patterns; (d) FTIR analysis; (e) optical properties; (f) emission spectra with varying excitation wavelengths.

The XRD pattern of CS, CN, CNCD, and CS/CNCD composite hydrogels is shown in Figure 2c. $\mathrm{CN}$ exhibited three peaks at $15.1^{\circ}, 16.3^{\circ}$, and $22.6^{\circ}$, assigned to $(101),(10 \overline{1})$, and (002) planes, respectively, indicating the crystallographic form of cellulose I [21]. These typical cellulose peaks were also observed in CNCD, indicating that the grafting of CD did not affect the crystalline structure of CN. CS had one broad peak at $19.8^{\circ}$ corresponding to the amorphous structure [22]. In the pattern of CS/CNCD composite hydrogel, these 
peaks cannot be found clearly, since CS and GD produced a cross-linked porous structure, and $\mathrm{CNCD}$ were wrapped in this produced cross-linked porous structure [23].

The FTIR spectra of CS, CN, CNCD, and CS/CNCD composite hydrogels are shown in Figure 2d. The broad absorption band at approximately $3348 \mathrm{~cm}^{-1}$ was attributed to the $\mathrm{O}-\mathrm{H}$ stretching vibration of $\mathrm{CN}$, but in the cases of $\mathrm{CS}, \mathrm{CNCD}$, and $\mathrm{CS} / \mathrm{CNCD}$ composite hydrogels, this band was also associated with the $\mathrm{N}-\mathrm{H}$ stretching vibration. In addition, an obvious characteristic band $\left(1048 \mathrm{~cm}^{-1}\right)$ closely related to cellulose species was found in both CN and CNCD [24]. The appearance of an absorption band at $1649 \mathrm{~cm}^{-1}$ associated with - $\mathrm{CO}-\mathrm{NH}$ - stretching vibrations confirmed that $\mathrm{CD}$ was grafted onto the $\mathrm{CN}$ [19]. Furthermore, the FTIR spectrum of CS/CNCD composite hydrogel contained several bands that differed from those of CS and CNCD. The broad absorption peak at $1646 \mathrm{~cm}^{-1}$ was specifically associated with $-\mathrm{C}=\mathrm{C}-\mathrm{N}=\mathrm{C}-$ stretching vibrations, indicating that crosslinking occurred to construct a new structure containing $-\mathrm{C}=\mathrm{C}-\mathrm{N}=\mathrm{C}-[25]$.

The optical properties of CS/CNCD composite hydrogel were investigated. The UV-vis spectrum exhibited two peaks at approximately $282 \mathrm{~nm}$ and $344 \mathrm{~nm}$ (Figure 2e), which could have originated from $n-\pi^{*}$ and $\pi-\pi^{*}$ transitions of CD [26]. The maximum emission peak of CS/CNCD composite hydrogel was centered at $478 \mathrm{~nm}$ under the $390 \mathrm{~nm}$ excitation. When $\lambda_{\text {ex }}$ moved from 345 to $435 \mathrm{~nm}$, the $\lambda_{\text {em }}$ shifted from 449 to $541 \mathrm{~nm}$ (Figure 2f). The emissions of a CS/CNCD composite hydrogel with excitation-dependent properties were confirmed, which contributed to the surface state influencing the $C D$ bandgap [27]. Moreover, the fluorescent stability of the CS/CNCD composite hydrogel was studied for different time periods, and the results are presented in Figure S5. The fluorescence and quantum yield (QY) were almost unchanged. Hence, this hydrogel had the potential for fluorescent probe.

\subsection{Sorption of $\mathrm{Cr}(V I)$}

The $\mathrm{pH}$ is a crucial parameter for $\mathrm{Cr}(\mathrm{VI})$ sorption. Hence, the sorption process is investigated at $\mathrm{pH} 1-6$ (Figure 3a). As the $\mathrm{pH}$ was 1 , the $Q_{\mathrm{e}}$ of the CS/CNCD composite hydrogel was low. According to Zeta potentials of the CS/CNCD composite hydrogel at different $\mathrm{pHs}$ (Figure S6), this hydrogel carried positive charges in the studied region. The $\mathrm{Cr}(\mathrm{VI})$ existed mainly as $\mathrm{H}_{2} \mathrm{CrO}_{4}$. Hence, there was the only weaker electrostatic attraction [28]. The second region was in the $\mathrm{pH}$ scope of 2-6, thus the $Q_{\mathrm{e}}$ of the CS/CNCD composite hydrogel began to decline. The $\mathrm{Cr}(\mathrm{VI})$ existed primarily as $\mathrm{HCrO}_{4}{ }^{-}$, and this hydrogel was electrostatically attracted to $\mathrm{HCrO}_{4}{ }^{-}$[29]. Due to the electrostatic attraction, this hydrogel had good adsorption performance for $\mathrm{Cr}(\mathrm{VI})$. As the $\mathrm{pH}$ increased in this region, the deprotonation reduced the $Q_{\mathrm{e}}$. As a result, the $\mathrm{pH}$ value 2 was chosen as the optimal $\mathrm{pH}$.
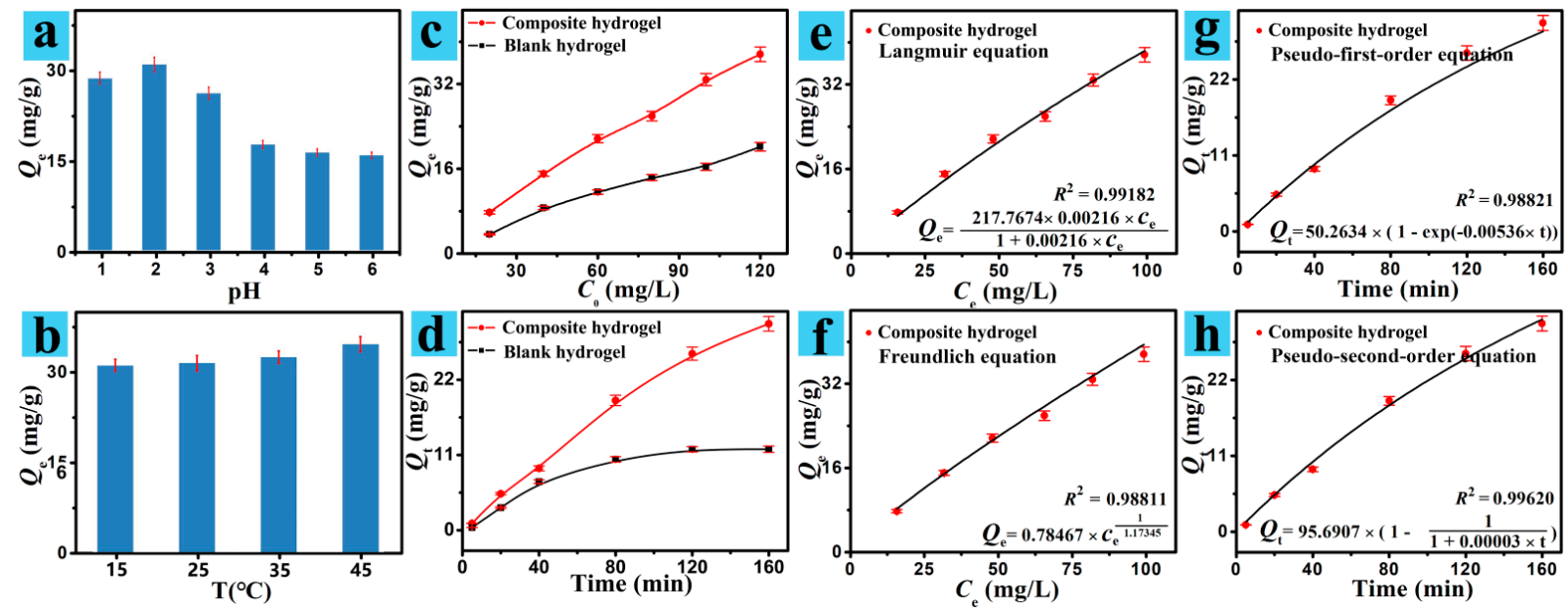

Figure 3. Effect of (a) $\mathrm{pH}$ and (b) temperature; (c,d) adsorption isotherm and sorption kinetic; (e,f) adsorption isotherms analyzed by two equations; (g,h) kinetics data analyzed by two equations. 
As a major parameter during the adsorption process, the effects of temperature on the adsorption process of the CS/CNCD composite hydrogel towards Cr(VI) ion (Figure 3b) were explored. The $Q_{e}$ of the CS/CNCD composite hydrogel increased, indicating that this sorption process may be endothermic [30]. It could be accounted for by the fact that the sorption capacity increases with increasing temperature. However, this increase was not so obvious in the temperature range of $15{ }^{\circ} \mathrm{C}-45^{\circ} \mathrm{C}$. Taking practical application into consideration, $25^{\circ} \mathrm{C}$ was used for the subsequent experiments.

The initial concentration of $\mathrm{Cr}(\mathrm{VI})$ ion can have an impact on the adsorption. As shown in Figure 3c, the $Q_{e}$ of the CS/CNCD composite hydrogel and the blank hydrogel increased significantly with increasing concentration. Origin 7.0 software was used to perform leastsquares curve fittings using the Langmuir and Freundlich equations. Fitting results are plotted in Figure 3e,f. The experimental data agreed well with the Langmuir equation with higher $\mathrm{R}^{2}$ (0.99182), showing this sorption onto the CS/CNCD composite hydrogel to be monolayer [31]. In addition, a maximum adsorption capacity $\left(Q_{\mathrm{m}}\right)$ of $217.8 \mathrm{mg} / \mathrm{g}$ was obtained. Some researchers also created adsorbents for $\mathrm{Cr}(\mathrm{VI})$, the majority of which had lower $Q_{\mathrm{m}}$, as shown in Table 1 .

Table 1. Maximum sorption capacities in comparison with previous work.

\begin{tabular}{cccc}
\hline Sample & Heavy Metals & $\boldsymbol{Q}_{\mathbf{m}} \mathbf{( \mathbf { m g } / \mathbf { g } )}$ & References \\
\hline Tetraethylenepentamine crosslinked chitosan & $C r(V I)$ & 150.5 & {$[32]$} \\
oligosaccharide hydrogel & $C r(V I)$ & 93.0 & {$[33]$} \\
Chitosan-based hydrogel & $C r(V I)$ & 27.3 & {$[34]$} \\
$\mathrm{Fe}_{3} \mathrm{O}_{4}$ NPs/CS/glyoxal hydrogel & $C r(V I)$ & 5.46 & {$[35]$} \\
Activated carbon & $C r(V I)$ & 2.2 & {$[36]$} \\
Magnetic chitosan/PVA hydrogel & $C r(V I)$ & 66.9 & {$[37]$} \\
Chitosan composite hydrogel & $C r(V I)$ & 78.0 & {$[38]$} \\
Chitosan/montmorillonite composite hydrogel & $C r(V I)$ & 66.1 & {$[39]$} \\
HPAM-chitosan gel bead & $C r(V I)$ & 217.8 & This work \\
The CS/CNCD composite hydrogel & & &
\end{tabular}

$\mathrm{Cr}(\mathrm{VI})$ adsorption as a function of contact time was further observed (Figure 3d). Compared with the blank hydrogel, the adsorption capacities of the CS/CNCD composite hydrogel were high in the same amount of contact time. The pseudo-first-order kinetic equation and the pseudo-second-order kinetic equation are two common mathematical models used to describe the sorption kinetics of the CS/CNCD composite hydrogel. The least-squares curve fittings were performed by Origin 7.0 software with these two equations [40]. Fitting results are plotted in Figure 3g,h. The experimental data were better fitted with a pseudo-second-order equation with a higher $\mathrm{R}^{2}(0.99620)$, indicating that chemical sorption was controlling the sorption onto the CS/CNCD composite hydrogel.

Reusability is an important criterion for a material to be applicable in practical purposes. Hence, the adsorption-desorption cycles were taken using the eluent of $\mathrm{HCl}$ solution $(1 \mathrm{~mol} / \mathrm{L})$; the results are shown in Figure S7. It was found that the CS/CNCD composite hydrogel retained $81.0 \%$ of its initial $Q_{e}$ after four cycles. As a result, the CS/CNCD composite hydrogel could be reused and recycled.

\subsection{Fluorescence Detection}

When the CS/CNCD composite hydrogel was challenged with coexisting metal ions $\left(\mathrm{Na}^{+}, \mathrm{Hg}^{2+}, \mathrm{Cr}^{6+}, \mathrm{Ba}^{2+}, \mathrm{Pb}^{2+}, \mathrm{Sr}^{2+}, \mathrm{Cu}^{2+}, \mathrm{Cr}^{3+}\right.$, and $\left.\mathrm{Al}^{3+}\right)$, the selectivity, fluorescence change, and QY were investigated. As shown in Figure $4 a, b, C r(V I)$ could cause significant fluorescence quenching of the CS/CNCD composite hydrogel, whereas other metal ions caused only minor quenching. Moreover, a good selectivity of the CS/CNCD composite hydrogel towards $\mathrm{Cr}(\mathrm{VI})$ over other anions was observed in Figure S8. These results display the excellent selectivity of the CS/CNCD composite hydrogel towards Cr(VI). 


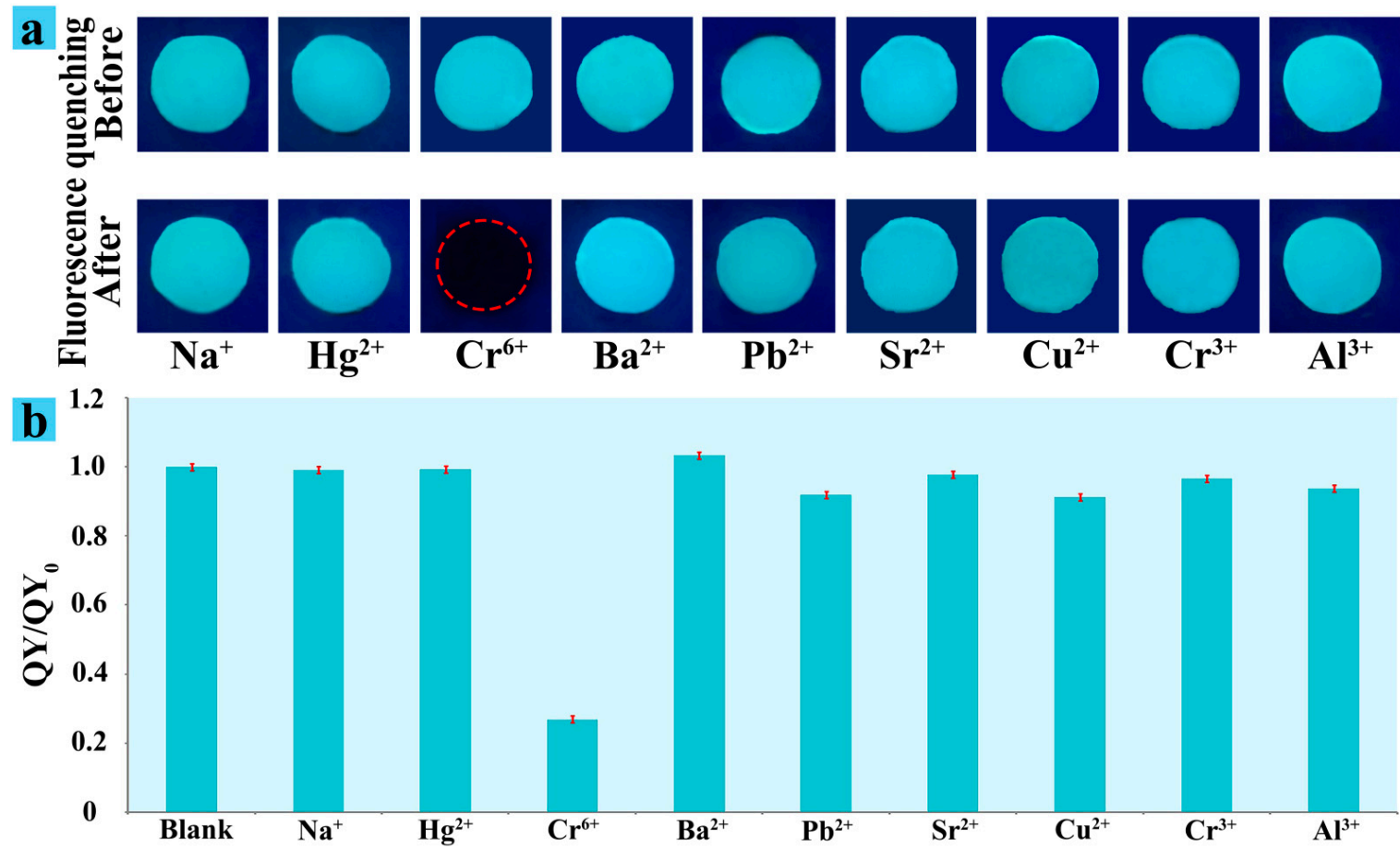

Figure 4. Fluorescence change (a) and QY (b) of CS/CNCD composite hydrogel with different heavy metals.

For the sensitivity study, various concentrations of $\mathrm{Cr}(\mathrm{VI})$ were thoroughly mixed with the CS/CNCD composite hydrogel. The fluorescence quenching decreased with $\mathrm{Cr}(\mathrm{VI})$ concentration, as shown in Figure 5a. The inset plot demonstrated a linear relationship between QY and Cr(VI) concentration (10-100 mg/L). Moreover, the fluorescence emission spectrum of the CS/CNCD composite hydrogel mixing with a lower concentration of $\mathrm{Cr}(\mathrm{VI})$ solution was collected, as shown in Figure $5 \mathrm{~b}$. As expected, the intensity of the signature of the peak at $478 \mathrm{~nm}$ decreased as concentration increased. The inset plot demonstrated a clear linear relationship between the intensity ratio $\left(\mathrm{I}_{0} / \mathrm{I}\right)-1$ and $\mathrm{Cr}(\mathrm{VI})$ concentrations (0.1-1.0 $\mu \mathrm{g} / \mathrm{L})$, with a detection limit of $0.04 \mu \mathrm{g} / \mathrm{L}$. The analytical indices of this method (linear ranges of $0.1-1.0 \mu \mathrm{g} / \mathrm{L}$ and $10-100 \mathrm{mg} / \mathrm{L}$, with a detection limit of $0.04 \mu \mathrm{g} / \mathrm{L}$ ) were compared to those of the other reports. The comparison results listed in Table S1 clearly show that the CS/CNCD composite hydrogel had a lower detection limit.
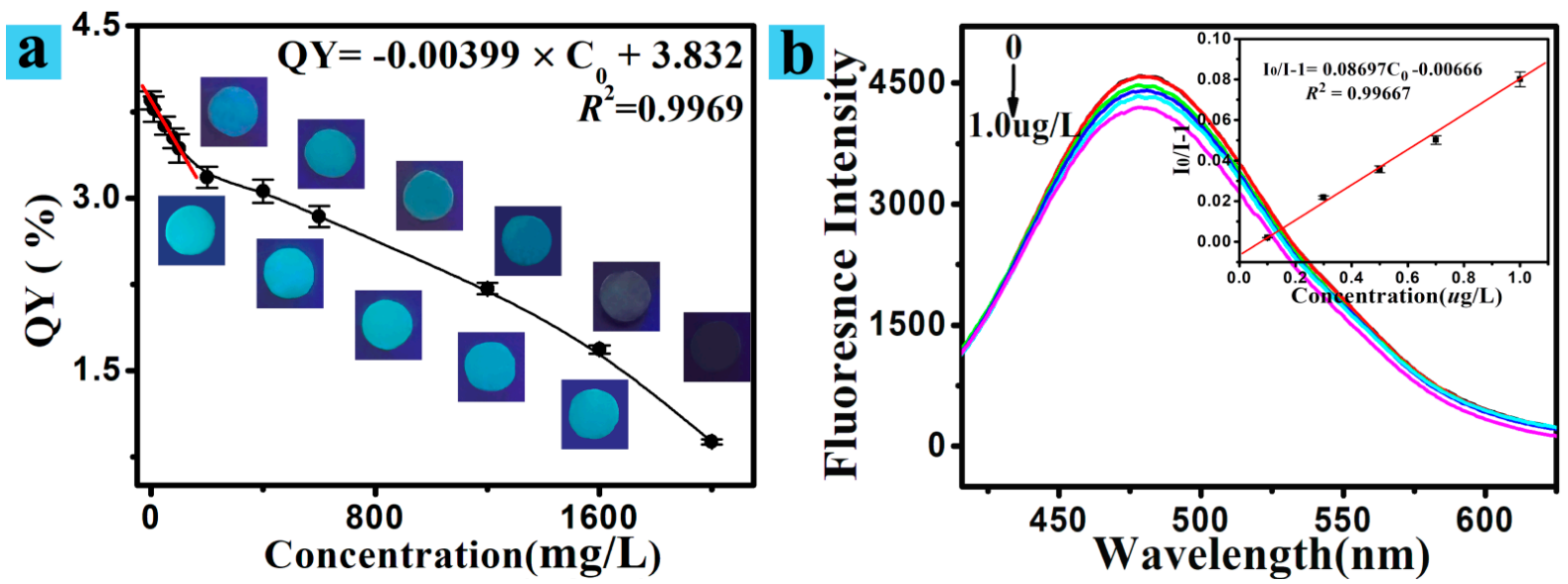

Figure 5. (a) Fluorescence change and QY of the CS/CNCD composite hydrogel mixed with different Cr(VI) concentrations; (b) fluorescent spectra of the CS/CNCD composite hydrogel mixed with lower $\mathrm{Cr}(\mathrm{VI})$ concentration, and the relationship between $\mathrm{I}_{0} / \mathrm{I}-1$ and concentration. 
The detection of $\mathrm{Cr}(\mathrm{VI})$ in tap and lake water was done by spiking different known concentrations of $\mathrm{Cr}(\mathrm{VI})$ solution to demonstrate the applicability of the CS/CNCD composite hydrogel. The average recoveries of $\mathrm{Cr}(\mathrm{VI})$ in spiked samples range from $99.17 \%$ to $101.01 \%$, with a relative standard deviation less than $4.95 \%$, as shown in Table S2. The results suggested that the CS/CNCD composite hydrogel had great potential for practical applications.

\section{5. $\mathrm{Cr}(\mathrm{VI})$ Sorption and Detection Mechanism}

The CS/CNCD composite hydrogel after adsorption processes was analyzed using FTIR in order to gain a better understanding of the sorption mechanism (Figure 6a). After sorption, the $3353 \mathrm{~cm}^{-1}$ peaks associated with $\mathrm{N}-\mathrm{H}$ and $\mathrm{O}-\mathrm{H}$ stretching vibrations and the $1559 \mathrm{~cm}^{-1}$ peak associated with -COO- stretching vibrations decreased. Furthermore, the $901 \mathrm{~cm}^{-1}$ peaks associated with $\mathrm{Cr}$ species increased [14]. It was indicated that the $\mathrm{Cr}(\mathrm{VI})$ sorption was closely related to hydroxyl, amino, and carboxyl groups.
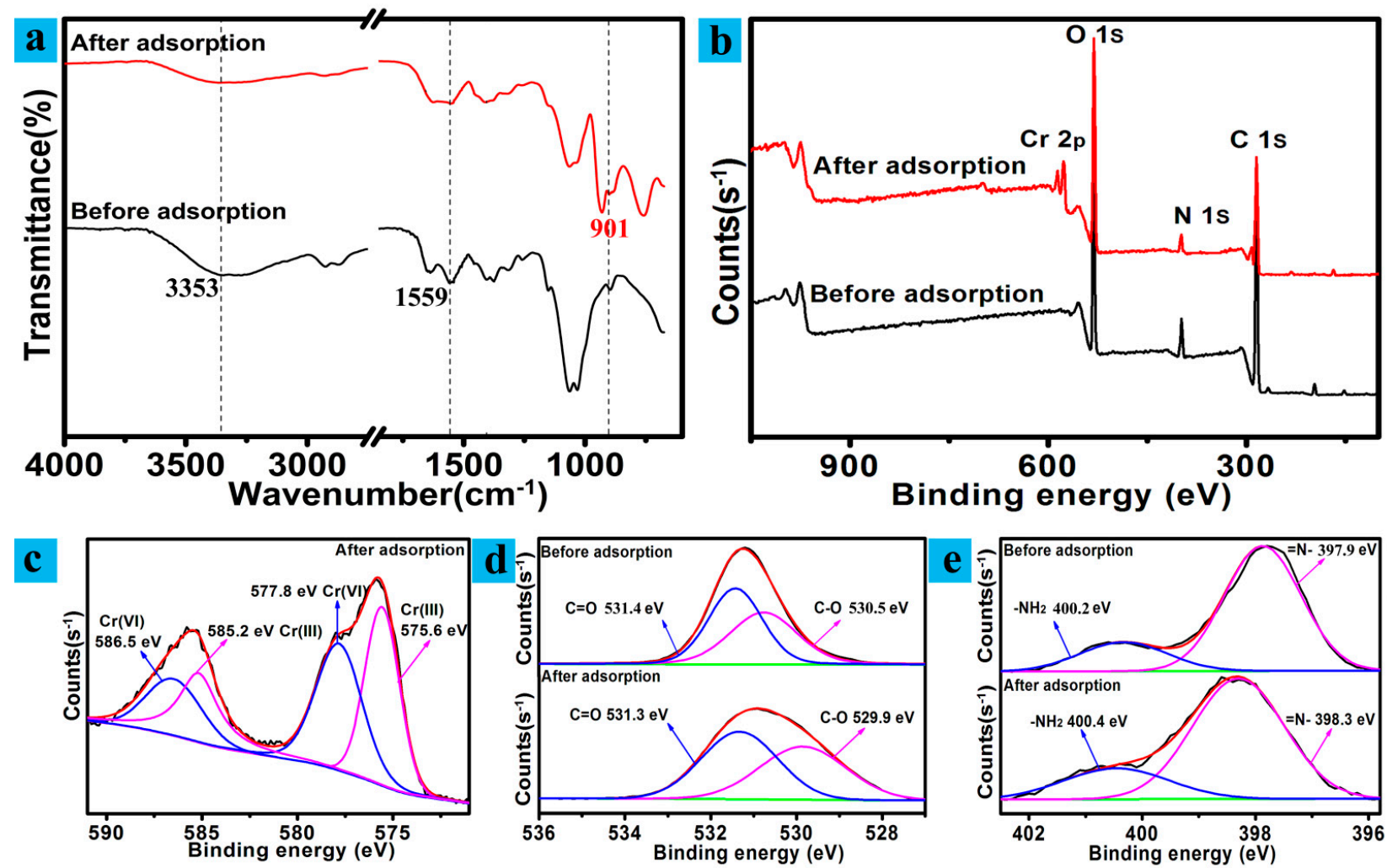

Figure 6. (a) FTIR spectra; (b) XPS total survey scan; (c-e) XPS spectra associated with Cr2p, O1s, and N1s.

To better understand the sorption mechanism, XPS analyses of the CS/CNCD composite hydrogel were performed. Total survey scans are displayed in Figure $6 \mathrm{~b}$. After sorption, four distinct peaks were observed: C1s (285 eV), N1s (400 eV), O1s (531 eV), and Cr2p $(585 \mathrm{eV})$. This result confirmed that the CS/CNCD composite hydrogel adsorbed $\mathrm{Cr}(\mathrm{VI})$ ion. Figure $6 \mathrm{c}$ shows the high-resolution $\mathrm{Cr} 2 \mathrm{p}$ spectra. It was shown that both $\mathrm{Cr} 2 \mathrm{p}_{3 / 2}$ and $\mathrm{Cr} 2 \mathrm{p}_{1 / 2}$ regions were split into two specific $\mathrm{Cr}(\mathrm{VI})$ and $\mathrm{Cr}(\mathrm{III})$ regions. The binding energies of 586.5 and $577.8 \mathrm{eV}$ were related to $\mathrm{Cr}(\mathrm{VI})$, while the binding energies of 585.2 and $575.6 \mathrm{eV}$ were related to $\mathrm{Cr}$ (III) [41]. The findings confirmed that a significant amount of $\mathrm{Cr}(\mathrm{VI})$ was reduced to $\mathrm{Cr}(\mathrm{III})$. Figure $6 \mathrm{~d}$ shows the high-resolution XPS results of O1s. The $\mathrm{O} 1$ s region was split into two components associated with $\mathrm{C}=\mathrm{O}$ and $\mathrm{C}-\mathrm{O}$. The integral area ratio of these two components grew, indicating that some $\mathrm{C}-\mathrm{O}$ groups could be oxidized into $\mathrm{C}=\mathrm{O}$ groups. Peaks at 398.3 and $400.4 \mathrm{eV}$ were observed in the high-resolution N1s spectrum (Figure 6e), which were related to imine $(=\mathrm{N}-)$ and amine $\left(-\mathrm{NH}_{2}\right)$ groups. The 
intensity of the signature from $-\mathrm{NH}_{2}$ was observed to rise after sorption, which indicated that the amino group participated in the sorption.

To elucidate the detection mechanism, UV-vis and fluorescence excitation spectra were obtained. The UV-Vis band of $\mathrm{Cr}(\mathrm{VI})$ overlapped with the CS/CNCD composite hydrogel (Figure S9a), implying that $\mathrm{Cr}(\mathrm{VI})$ detection could be due to the IFE. As shown in Figure S9b-d, the average fluorescence life of the CS/CNCD composite hydrogel $\left(\tau^{*}=5.9 \mathrm{~ns}\right)$ was very close to that of the CS/CNCD composite hydrogel adsorbed with $\mathrm{Cr}(\mathrm{VI})\left(\tau^{*}=6.0 \mathrm{~ns}\right)$, confirming that there was static quenching. The IFE and static quenching were used to power the detection mechanism.

According to the above-mentioned analytical results, the CS/CNCD composite hydrogel's $\mathrm{Cr}(\mathrm{VI})$ sorption and detection mechanism are as follows (Figure 7). $\mathrm{The} \mathrm{Cr}(\mathrm{VI})$ was first enriched into the interface via electric attraction. After that, $\mathrm{Cr}(\mathrm{VI})$ was gradually diffused to the inside along 3D porous structures. In this diffusion process, a considerable amount of $\mathrm{Cr}(\mathrm{VI})$ was reduced to $\mathrm{Cr}(\mathrm{III})$, then some of the $\mathrm{Cr}(\mathrm{III})$ ions interacted with the hydroxyl and carboxyl groups. Due to these specific structures, the CS/CNCD composite hydrogel had a high adsorption capacity. Concurrently, $\mathrm{Cr}(\mathrm{VI})$ was enriched into the CD via electric attraction, resulting in fluorescence quenching. As a result, the CS/CNCD composite hydrogel possessed sensitive detection capability.

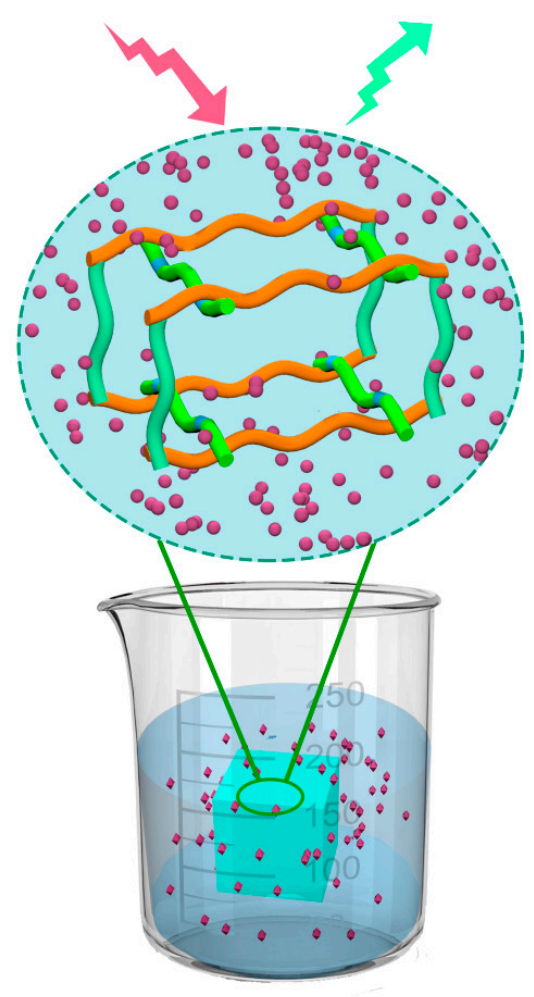

3D structure

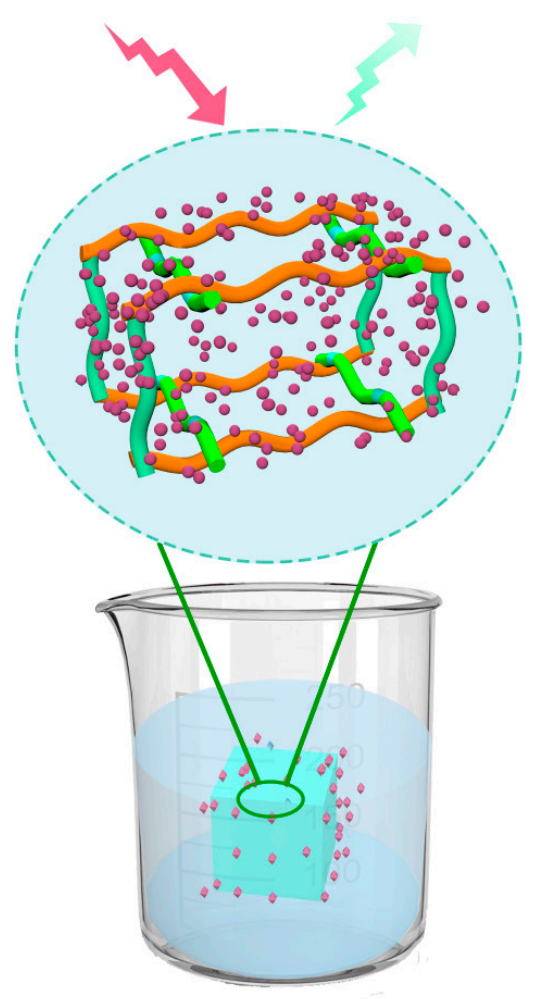

Hazardous Cr(VI)

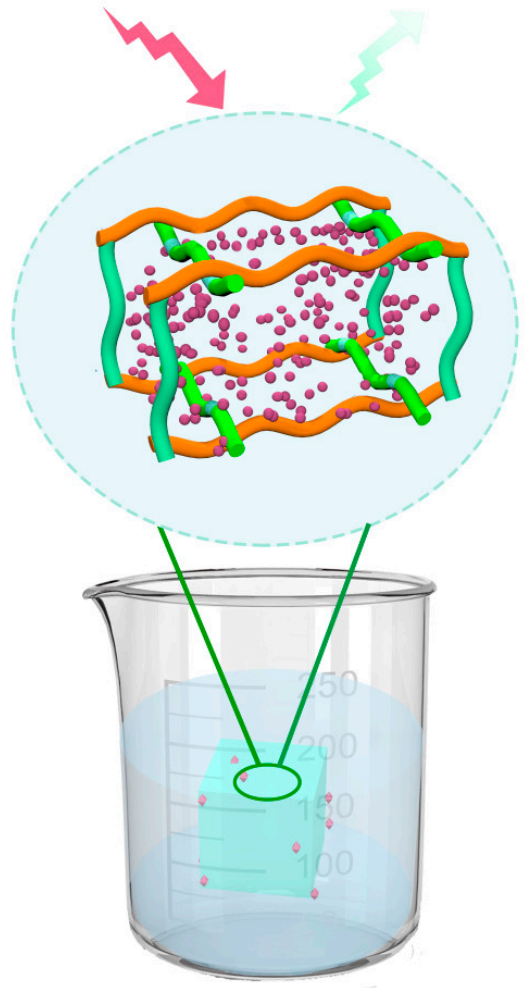

Hydrogel

Figure 7. Sorption and detection mechanism of the CS/CNCD composite hydrogel.

\subsection{Economic Feasibility}

The production cost is a critical factor in considering whether an adsorbent has potential. The net cost of producing an adsorbent typically includes the cost of raw material, reaction reagent, and operating reaction equipment [42]. Table S3 shows the total cost for preparing $1 \mathrm{~kg}$ of the CS/CNCD composite hydrogel. In comparison with the commercially activated carbon (AC), the CS/CNCD composite hydrogel was cheaper. 
Moreover, Table $\mathrm{S} 4$ shows the cost incurred in adsorbing $1 \mathrm{~g} \mathrm{Cr}(\mathrm{VI})$ using the CS/CNCD composite hydrogel compared to AC. According to this cost analysis, the cost of removing $1 \mathrm{~g} \mathrm{Cr}(\mathrm{VI})$ for the CS/CNCD composite hydrogel was only CNY 0.62, which was nearly 58.8 times less expensive than AC (CNY 36.48). The CS/CNCD composite hydrogel appears to be a promising adsorbent for $\mathrm{Cr}(\mathrm{VI})$ removal.

\section{Conclusions}

In this study, a novel hydrogel was prepared via CS incorporating CN modified with $\mathrm{CD}$, followed by crosslinking with GD with a strong sorption ability and sensitive detection ability for $\mathrm{Cr}(\mathrm{VI})$. The effects of various parameters on $\mathrm{Cr}(\mathrm{VI})$ sorption were investigated, including initial $\mathrm{pH}$, temperature, contact time, and initial concentration. The CS/CNCD composite hydrogel had high absorption properties, with a maximum adsorption capacity of $217.8 \mathrm{mg} / \mathrm{g}$. Meanwhile, this hydrogel was employed for selective and quantitative detection of $\mathrm{Cr}(\mathrm{VI})$ with linear ranges of $0.1-1.0 \mu \mathrm{g} / \mathrm{L}$ and $10-100 \mathrm{mg} / \mathrm{L}$, and a detection limit of $0.04 \mu \mathrm{g} / \mathrm{L}$. Furthermore, an economic analysis of this hydrogel was performed, which revealed that the cost of removing $1 \mathrm{~g} \mathrm{Cr}(\mathrm{VI})$ for the $\mathrm{CS} / \mathrm{CNCD}$ composite hydrogel was nearly 58.8 times lower than that of AC. All of this demonstrated that the developed hydrogel could be used to treat $\mathrm{Cr}(\mathrm{VI})$ wastewater.

Supplementary Materials: The following are available online at https: / www.mdpi.com/article / 10.3390 / polym13213788/s1, Figure S1: TEM image of the CDs, Figure S2: Fluorescence spectra (a) and fluorescence intensity (b) of a series of CNCD prepared by using different concentrations of CD solution, Figure S3: Maximum adsorption capacities for $\mathrm{Cr}(\mathrm{VI})$ of a series of hydrogels, Figure S4: Pore characteristics of the CS/CNCD composite hydrogel, Figure S5: Fluorescent stability of the CS/CNCD composite hydrogel, Figure S6: Zeta potentials of the CS/CNCD composite hydrogel, Figure S7: Adsorption-desorption cycles, Figure S8: Fluorescence change and QY of CS/CNCD composite hydrogel with different anions and $\mathrm{Cr}(\mathrm{VI})$, Figure S9: (a) UV-Vis absorption spectrum of $\mathrm{Cr}(\mathrm{VI})$ and excitation spectrum of the CS/CNCD composite hydrogel. (b) Fluorescence decay curves of the CS/CNCD composite hydrogel and the CS/CNCD composite hydrogel with adsorbed $\mathrm{Cr}(\mathrm{VI})$ ion. (c,d) Fluorescence decay curves fitted by second-order exponential functions, Table S1: Comparison of sensing performance of different sensors for $\mathrm{Cr}(\mathrm{VI})$ detection, Table S2: Recovery and RSD of $\mathrm{Cr}(\mathrm{VI})$ in tap and lake water samples, Table S3: Breakup and total cost for preparing $1 \mathrm{~kg}$ of CS/CNCD composite hydrogel, Table S4: Cost of adsorbent for the removal of $1 \mathrm{~g}$ of $\mathrm{Cr}(\mathrm{VI})$.

Author Contributions: Conceptualization, H.Z. and Z.H.; methodology, C.P.; software, L.D.; validation, H.Z., Z.H. and C.P.; formal analysis, H.Z.; writing-original draft preparation, H.Z.; writingreview and editing, H.Z.; visualization, Z.H.; supervision, S.L.; project administration, S.L.; funding acquisition, S.L. All authors have read and agreed to the published version of the manuscript.

Funding: This research was funded by the National Natural Science Foundation of China (grant number 31571802), and the Innovation and Entrepreneurship Training Program for College Students of Hunan Province (grant number s202010537034).

Institutional Review Board Statement: Not applicable.

Informed Consent Statement: Not applicable.

Data Availability Statement: All the data are available within the manuscript.

Conflicts of Interest: The authors declare no conflict of interest.

\section{References}

1. Godiya, C.B.; Cheng, X.; Li, D.; Chen, Z.; Lu, X. Carboxymethyl cellulose/polyacrylamide composite hydrogel for cascaded treatment/reuse of heavy metal ions in wastewater. J. Hazard. Mater. 2019, 364, 28-38. [CrossRef] [PubMed]

2. Park, S.; Shin, S.S.; Park, C.H.; Jeon, S.; Gwon, J.; Lee, S.; Kim, S.; Kim, H.; Lee, J. Poly(acryloyl hydrazide)-grafted cellulose nanocrystal adsorbents with an excellent Cr(VI) adsorption capacity. J. Hazard. Mater. 2020, 394, 122512. [CrossRef] [PubMed]

3. Sigroha, S.; Khatkar, A. Chitosan-A naturally derived antioxidant polymer with diverse applications. Curr. Org. Chem. 2017, 21, 333-341. [CrossRef] 
4. Pavithra, S.; Thandapani, G.; Sugashini, S.; Sudha, P.N.; Alkhamis, H.H.; Alrefaei, A.F.; Almutairi, M.H. Batch adsorption studies on surface tailored chitosan/orange peel hydrogel composite for the removal of $\mathrm{Cr}(\mathrm{VI})$ and $\mathrm{Cu}(\mathrm{II})$ ions from synthetic wastewater. Chemosphere 2021, 271, 129415. [CrossRef] [PubMed]

5. Hao, D.; Liang, Y. Adsorption of $\mathrm{Cu}^{2+}, \mathrm{Cd}^{2+}$ and $\mathrm{Pb}^{2+}$ in wastewater by modified chitosan hydrogel. Environ. Technol. 2020. [CrossRef]

6. Liu, Y.; Hu, L.; Yao, Y.; Su, Z.; Hu, S. Construction of composite chitosan-glucose hydrogel for adsorption of $\mathrm{Co}^{2+}$ ions. Int. J. Biol. Macromol. 2019, 139, 213-220. [CrossRef]

7. Tang, S.; Yang, J.; Lin, L.; Peng, K.; Chen, Y.; Jin, S.; Yao, W. Construction of physically crosslinked chitosan/sodium alginate/calcium ion double-network hydrogel and its application to heavy metal ions removal. Chem. Eng. J. 2020, $393,124728$. [CrossRef]

8. Isogai, A.; Saito, T.; Fukuzumi, H. TEMPO-oxidized cellulose nanofibers. Nanoscale 2011, 3, 71-85. [CrossRef]

9. Li, Y.; Miao, P.; Zhou, W.; Gong, X.; Zhao, X. N-doped carbon-dots for luminescent solar concentrators. J. Mater. Chem. A. 2017, 5, 21452-21459. [CrossRef]

10. Ganguly, S.; Das, P.; Itzhaki, E.; Hadad, E.; Gedanken, A.; Margel, S. Microwave-synthesized polysaccharide-derived carbon dots as therapeutic cargoes and toughening agents for elastomeric gels. ACS Appl. Mater. Interfaces 2020, 12, 51940-51951. [CrossRef]

11. Zhang, Y.; Li, C.; Sun, L.; Zhang, J.; Yang, X.; Ma, H. Defects coordination triggers red-shifted photoluminescence in carbon dots and their application in ratiometric $\mathrm{Cr}(\mathrm{VI})$ sensing. Microchem. J. 2021, 169, 106552. [CrossRef]

12. Ganguly, S.; Das, P.; Das, S.; Ghorai, U.; Bose, M.; Ghosh, S.; Mondal, M.; Das, A.K.; Banerjee, S.; Das, N.C. Microwave assisted green synthesis of Zwitterionic photolumenescent $\mathrm{N}$-doped carbon dots: An efficient 'on-off' chemosensor for tracer $\mathrm{Cr}(+6)$ considering the inner filter effect and nano drug-delivery vector. Colloid. Surf. A 2019, 579, 123604. [CrossRef]

13. Yang, X.; Hu, D.; Zhang, P.; Ding, H.; Ji, Y.; Zou, H.; Li, B.; Wei, J.; Wei, X. Integrated carbon dots-matrix structures: An efficient strategy for high-performance electric double layer capacitors. ACS Appl. Energy Mater. 2020, 3, 4958-4964. [CrossRef]

14. Luo, Q.; Yuan, H.; Zhang, M.; Jiang, P.; Liu, M.; Xu, D.; Guo, X.; Wu, Y. A 3D porous fluorescent hydrogel based on amino-modified carbon dots with excellent sorption and sensing abilities for environmentally hazardous Cr(VI). J. Hazard. Mater. 2021, 401, 123432. [CrossRef] [PubMed]

15. Ujihara, M.; Hsu, M.; Liou, J.; Imae, T. Hybridization of cellulose nanofiber with amine-polymers and its ability on sick house syndrome gas decomposition. J. Taiwan Inst. Chem. E 2018, 92, 106-111. [CrossRef]

16. Zhu, S.; Meng, Q.; Wang, L.; Zhang, J.; Song, Y.; Jin, H.; Zhang, K.; Sun, H.; Wang, H.; Yang, B. Highly photoluminescent carbon dots for multicolor patterning, sensors, and bioimaging. Angew. Chem. Int. Ed. 2013, 52, 3953-3957. [CrossRef]

17. $\mathrm{Wu}, \mathrm{B}$.; Zhu, G.; Dufresne, A.; Lin, N. Fluorescent aerogels based on chemical crosslinking between nanocellulose and carbon dots for optical sensor. ACS Appl. Mater. Interfaces 2019, 11, 16048-16058. [CrossRef]

18. Han, X.; Liu, Y.; Xiong, L.; Huang, H.; Zhang, Q.; Li, L.; Yu, X.; Wei, L. Facile assembly of Polyaniline/Graphene oxide composite hydrogels as adsorbent for Cr(VI) removal. Polym. Compos. 2019, 402, E1777-E1785. [CrossRef]

19. Luo, Q.; Huang, X.; Luo, Y.; Yuan, H.; Ren, T.; Li, X.; Xu, D.; Guo, X.; Wu, Y. Fluorescent chitosan-based hydrogel incorporating titanate and cellulose nanofibers modified with carbon dots for adsorption and detection of $\mathrm{Cr}(\mathrm{VI})$. Chem. Eng. J. 2021, 407, 127050. [CrossRef]

20. Demirci, N.; Demirel, M.; Dilsiz, N. Surface modification of PVC film with allylamine plasma polymers. Adv. Polym. Technol. 2014, 33, 21435. [CrossRef]

21. Han, J.; Zhou, C.; Wu, Y.; Liu, F.; Wu, Q. Self-assembling behavior of cellulose nanoparticles during freeze-drying: Effect of suspension concentration, particle size, crystal structure, and surface charge. Biomacromolecules 2013, 14, 1529-1540. [CrossRef] [PubMed]

22. Skwarczynska, A.; Kaminska, M.; Owczarz, P.; Bartoszek, N.; Walkowiak, B.; Modrzejewska, Z. The structural (FTIR, XRD, and XPS) and biological studies of thermosensitive chitosan chloride gels with -glycerophosphate disodium. J. Appl. Polym. Sci. 2018, 135, 46459. [CrossRef]

23. Li, S.; Wang, X.; An, Q.; Xiao, Z.; Zhai, S.; Cui, L.; Li, Z. Upon designing carboxyl methylcellulose and chitosan-derived nanostructured sorbents for efficient removal of Cd(II) and Cr(VI) from water. Int. J. Biol. Macromol. 2020, 143, 640-650. [CrossRef] [PubMed]

24. Luo, Q.; Ren, T.; Lei, Z.; Huang, Y.; Huang, Y.; Xu, D.; Wan, C.; Guo, X.; Wu, Y. Non-toxic chitosan-based hydrogel with strong adsorption and sensitive detection abilities for tetracycline. Chem. Eng. J. 2022, 427, 131738. [CrossRef]

25. Geng, Z.; Zhang, H.; Xiong, Q.; Zhang, Y.; Zhao, H.; Wang, G. A fluorescent chitosan hydrogel detection platform for the sensitive and selective determination of trace mercury(II) in water. J. Mater. Chem. A. 2015, 3, 19455-19460. [CrossRef]

26. Song, S.; Liang, F.; Li, M.; Du, F.; Dong, W.; Gong, X.; Shuang, S.; Dong, C. A label-free nano-probe for sequential and quantitative determination of $\mathrm{Cr}(\mathrm{VI})$ and ascorbic acid in real samples based on $\mathrm{S}$ and $\mathrm{N}$ dual-doped carbon dots. Spectrochim. Acta A 2019, 215, 58-68. [CrossRef]

27. Li, W.; Wang, S.; Li, Y.; Ma, C.; Huang, Z.; Wang, C.; Li, J.; Chen, Z.; Liu, S. One-step hydrothermal synthesis of fluorescent nanocrystalline cellulose/carbon dot hydrogels. Carbohydr. Polym. 2017, 175, 7-17. [CrossRef]

28. Maity, J.; Ray, S.K. Enhanced adsorption of $\mathrm{Cr}(\mathrm{VI})$ from water by guar gum based composite hydrogels. Int. J. Biol. Macromol. 2016, 89, 246-255. [CrossRef] 
29. Abdel-Halim, E.S.; Al-Deyab, S.S. Hydrogel from crosslinked polyacrylamide/guar gum graft copolymer for sorption of hexavalent chromium ion. Carbohydr. Polym. 2011, 86, 1306-1312. [CrossRef]

30. Yuan, H.; Yang, G.; Luo, Q.; Xiao, T.; Zuo, Y.; Guo, X.; Xu, D.; Wu, Y. A 3D net-like structured fluorescent aerogel based on carboxy-methylated cellulose nanofibrils and carbon dots for a highly effective adsorbent and sensitive optical sensor of $\mathrm{Cr}(\mathrm{VI})$. Environ. Sci. Nano 2020, 7, 773-781. [CrossRef]

31. Zhou, G.; Luo, J.; Liu, C.; Chu, L.; Ma, J.; Tang, Y.; Zeng, Z.; Luo, S. A highly efficient polyampholyte hydrogel sorbent based fixed-bed process for heavy metal removal in actual industrial effluent. Water Res. 2016, 89, 151-160. [CrossRef] [PubMed]

32. Mei, J.; Zhang, H.; Li, Z.; Ou, H. A novel tetraethylenepentamine crosslinked chitosan oligosaccharide hydrogel for total adsorption of $\mathrm{Cr}(\mathrm{VI})$. Carbohydr. Polym. 2019, 224, 115154. [CrossRef]

33. Vilela, P.B.; Dalalibera, A.; Duminelli, E.C.; Becegato, V.A.; Paulino, A.T. Adsorption and removal of chromium (VI) contained in aqueous solutions using a chitosan-based hydrogel. Environ. Sci. Pollut. Res. 2019, 26, 28481-28489. [CrossRef] [PubMed]

34. Mirabedini, M.; Kassaee, M.Z.; Poorsadeghi, S. Novel magnetic chitosan hydrogel film, Cross-Linked with glyoxal as an efficient adsorbent for removal of toxic Cr(VI) from water. Arab. J. Sci. Eng. 2017, 42, 115-124. [CrossRef]

35. Adebayo, G.B.; Adegoke, H.I.; Fauzeeyat, S. Adsorption of $\mathrm{Cr}(\mathrm{VI})$ ions onto goethite, activated carbon and their composite: Kinetic and thermodynamic studies. Appl. Water Sci. 2020, 10, 213. [CrossRef]

36. Yi, N.; Wu, Y.; Wei, J.; Zhang, S.; Ji, P. Adsorption of the low concentration Cr (VI) on magnetic chitosan/PVA hydrogel beads. Fresen. Environ. Bull. 2016, 25, 2174-2182.

37. Tekay, E.; Sen, S.; Aydinoglu, D.; Nugay, N. Biosorbent immobilized nanotube reinforced hydrogel carriers for heavy metal removal processes. $e$-Polymers 2016, 16, 15-24. [CrossRef]

38. Tekay, E.; Aydinoglu, D.; Sen, S. Effective adsorption of $\mathrm{Cr}(\mathrm{VI})$ by high strength chitosan/montmorillonite composite hydrogels involving spirulina Biomass/Microalgae. J. Polym. Environ. 2019, 27, 1828-1842. [CrossRef]

39. Kuang, W.; Tan, Y.; Fu, L. Adsorption kinetics and adsorption isotherm studies of chromium from aqueous solutions by HPAM-chitosan gel beads. Desalin. Water Treat. 2012, 45, 222-228. [CrossRef]

40. Zhou, G.; Luo, J.; Liu, C.; Chu, L.; Crittenden, J. Efficient heavy metal removal from industrial melting effluent using fixed-bed process based on porous hydrogel adsorbents. Water Res. 2018, 131, 246-254. [CrossRef]

41. Li, R.; Liang, W.; Li, M.; Jiang, S.; Huang, H.; Zhang, Z.; Wang, J.J.; Awasthi, M.K. Removal of Cd(II) and Cr(VI) ions by highly cross-linked thiocarbohydrazide-chitosan gel. Int. J. Biol. Macromol. 2017, 104, 1072-1081. [CrossRef] [PubMed]

42. Roman, S.; Valente Nabais, J.M.; Ledesma, B.; Gonzalez, J.F.; Laginhas, C.; Titirici, M.M. Production of low-cost adsorbents with tunable surface chemistry by conjunction of hydrothermal carbonization and activation processes. Microporous Mesoporous Mater. 2013, 165, 127-133. [CrossRef] 